\title{
Mechanical treatment of waste water from fat substances in the ultrasonic field
}

\author{
Pavel Vikulin and Vera Vikulina
}

Moscow State University of Civil Engineering, Yaroslavskoe shosse, 26, Moscow, 129337, Russia

\begin{abstract}
The use of ultrasonic vibrations in the technology of waste water purification from fat substances is part of the scientific problem of using in this area of knowledge of physical methods, which include: ultrasonic, magnetic, high-frequency, roentgen, ultraviolet and other. Currently, ultrasonic vibrations are widely used in various industries: metallurgy, chemical, mechanical engineering, medicine and other. The wide use of ultrasound is explained by the variety of phenomena occurring in the ultrasonic field. Ultrasonic vibrations initiate dispersion and emulsification of substances, contribute to coagulation and degassing, affect the processes of crystallization and dissolution; it is known that ultrasound causes a variety of chemical transformations of the substance, including oxidation, reduction, polymerization and so on. The explanation of these phenomena is found in a variety of ultrasonic effects: cavitation, shock waves, micro-flows, acoustic wind and so on. The schemes of ultrasonic generators are continuously improved, works on the development of methods of using ultrasound and on the search for new areas of its application are progressing. Under these conditions, the question of the possibility of using ultrasound for the treatment of waste water from fat is relevant. However, the theoretical side of this issue is a little-studied object.
\end{abstract}

\section{Introduction}

Relevance of the topic. Application of the ultrasonic field in cavitation mode is currently used in various industries - metallurgical, chemical, nuclear power. The widespread introduction of ultrasound in various technologies due to physical and chemical effects on the substance. The development of a theoretical model and method for calculating the effect of ultrasound in cavitation mode on the release of fatty substances from the liquid is an actual topic of application of physical methods in industrial technologies.

Basic theoretical background.

Ultrasound - elastic vibrations. Ultrasonic waves of high intensity are described only by the laws of nonlinear acoustics. The propagation of ultrasonic waves in liquids is accompanied by acoustic flow, condensation, and medium discharge. Acoustic cavitation is among the important nonlinear phenomena in an ultrasonic field. Investigation of the mechanism of the action of acoustic fields on the matter is complicated by the fact that there are simultaneously different processes in the ultrasonic field that can have mutual influence. Therefore, it is difficult to describe the experimental results. At the present time, theoretical 
grounds for the use of physical methods, in particular, ultrasonic vibrations, in water technologies are being developed. The use of an ultrasonic field to intensify oxidationreduction processes in an aqueous medium, the precipitation of coarsely dispersed impurities expands the area of possible use of this physical method [1-4]. Sound, as a physical phenomenon, is characterized by the sound pressure, the density of sound energy, the flow of sound energy, the level of intensity (power) of sound. Liquids in the static state do not have a shear viscosity and are incapable of withstanding and transmitting any tangential stresses. Therefore, only longitudinal waves propagate in liquids and gases, in which the direction of the tangential motions of the particles coincides with the direction of propagation of the waves. The propagation velocity depends on the density of the medium $\rho$ and the adiabatic compressibility coefficient $\beta \mathrm{c}$ and is calculated from the formula:

$$
c=\sqrt{\frac{1}{\rho \cdot \beta_{\mathrm{c}}}} .
$$

The physical nature of propagation of elastic waves is described by equation

$$
y=A \cdot \sin \omega \cdot t
$$

where $A$ - bias amplitude;

$\omega$ - cyclic frequency;

$t$ - a period of time.

Acoustic vibrations in the medium create additional pressure [5-7]. The sound wave, passing through the liquid, creates zones of compression and depletion, changing places in each half-period of the passage of the wave. This gives rise to a sign-variable pressure, $P$, which can be determined from formula:

$$
P=\sqrt{\rho \cdot c \cdot I} \cdot 4.6 \cdot 10^{-3} .
$$

Sound pressure is a variable that varies periodically. At a given point in the medium, during a period, the pressure $P$ changes from a maximum to zero and then rises again to a maximum value, and corresponds to a description of harmonic oscillations:

$$
P=P_{\max } \cdot \sin \omega \cdot t
$$

where $P_{\max }$ is the maximum sound pressure (pressure amplitude), defined by formula

$$
P_{\max }=\omega \cdot c \cdot \rho \cdot A,
$$

where $c$ - speed of sound.

When ultrasound propagates in the medium, part of its energy is absorbed, and the medium heats up. Absorption of acoustic energy is due to the frequency of sound, viscosity, thermal conductivity and wave resistance of the medium, i.e. the product of the density of the medium $\rho$ and the speed of sound.

The relationship between the sound pressure developed in the medium and the wave impedance expresses the vibrational velocity. $[8,9]$

The value of the wave impedance of the medium is determined by the ratio of the sound pressure in the traveling plane wave to the vibrational velocity of the particles of the medium $V$ 


$$
\frac{P}{V}=\mathrm{r} \Psi
$$

Vibrational velocity and sound pressure do not depend on frequency, the amplitude of the oscillations is inversely proportional, and the acceleration is directly proportional to the frequency. Usually the amplitudes of the vibrational velocities are many orders of magnitude lower than the sound velocity in the unperturbed liquid $\left(10^{3} \mathrm{~m} / \mathrm{s}\right)$. For moderate acoustic fields, the sound pressure usually does not exceed $1 \mathrm{MPa}$, but the pressure gradient, especially at high frequencies, can reach large values.

The amplitude of the acceleration of liquid particles in the field of ultrasonic waves is great; they can exceed the acceleration of free fall by several orders of magnitude. In technology such accelerations are achieved only in special ultracentrifuges. Also taking into account the fact that such values of acceleration change sign twice during the period, one can consider ultrasonic waves a very powerful and peculiar physical factor affecting the substance even in the absence of nonlinear effects. The main physicochemical and chemical effects that arise in a liquid under the action of acoustic fields are due mainly to nonlinear effects, of which the most important is cavitation [10, 11, 12, 13].

One of the characteristic features of ultrasonic cavitation is that it is a peculiar and effective mechanism of local concentration of relatively low average energy of the acoustic field in very small volumes, which leads to the creation of exceptionally high energy densities. The detailed mechanism of this effect is not yet completely clear.

In connection with the fact that the phenomenon of cavitation represents a great theoretical and practical interest in the basic physicochemical problems of cavitation, considerable attention will be paid subsequently. The wave resistance of the medium is a very important characteristic that determines the conditions for radiation, absorption of acoustic vibrations, their reflection, refraction, etc.

Particles of the elastic medium in which ultrasonic waves propagate vibrate and therefore possess kinetic and potential energy. The amount of energy transferred by sound vibrations per second through an area of $1 \mathrm{sm}^{2}$, which is perpendicular to the direction of their propagation, characterizes the intensity of sound and is determined by the formula:

$$
I=E_{1} \cdot c
$$

where $E_{1}$ - energy density.

The density of sound energy at each point varies with time. The average value of the energy density at a given point is determined by the formula

$$
E_{1}=\frac{1}{2} \cdot A^{2} \cdot \omega^{2} \cdot \rho
$$

Transforming equation (7) and equation (8) we obtain expression (9)

$$
I=\frac{1}{2} \cdot A^{2} \cdot \omega^{2} \cdot \rho \cdot c
$$

In the propagation of sound waves in a liquid medium, the intensity of sound $I$ decreases with increasing distance from the source of radiation from equation:

$$
I=I_{0} \cdot e^{-2 \alpha X},
$$

where $I_{0}-$ sound intensity at $X=0$,

$\alpha-$ absorption coefficient. 
The absorption coefficient (absorption coefficient) depends on the physical properties of the substance, is a characteristic parameter of the substance, and also depends on external conditions (temperature, pressure) and the frequency of oscillations. Although the physical nature of ultrasound and the basic laws describing its propagation are the same as for sound waves of any frequency range, it has a number of specific features. These features are due to its relatively high frequencies and correspondingly small wavelengths [14-16].

In the high-intensity ultrasonic field, significant acoustic currents develop, the velocity of which is usually small in comparison with the vibrational velocity of the particles [1719]. The most important nonlinear effect in the ultrasonic field is cavitation-the appearance of a mass of pulsating bubbles filled with steam, gas or a mixture of them in the liquid [20]. The complex motion of bubbles, their slamming, merging with each other, etc generate compression pulses in the liquid (micro-shock waves) and micro-flows, cause local heating of the medium, ionization. These effects have an impact on the substance: there is a destruction of solid particles in the liquid, (cavitation erosion), there is a mixing of the liquid, initiated or accelerated by various physical and chemical processes.

By changing the conditions of cavitation, it is possible to strengthen or weaken various cavitation effects, for example, with the increase in the frequency of ultrasound, the role of micro-flows increases and cavitation erosion decreases, with the increase in hydrostatic pressure in the liquid, the role of micro-shock effects increases.

Various applications of ultrasonic vibrations, which use different features, can be divided into three directions. The first is connected with obtaining information by means of ultrasonic waves, the second - with active influence on substance and the third - with processing and transmission of signals [21].

The variety of industrial plants and their technologies generate the same variety of waste water compositions.

The introduction of the physical method-ultrasonic cavitation-requires theoretical developments.

The purpose of the work is to create a model of the process of separation of fat substances from wastewater in an ultrasonic field. The task of the paper is obtaining a mathematical equation describing the effect of acoustic cavitation on the effects of separation of pollutants in the water environment.

\section{Results}

In order to mathematically describe the process that occurs in the suspension when the ultrasonic field is applied, we assume that ultrasonic vibrations in the cavitation mode release the fat particle, destroying the film from the impurities, thereby contributing to the release of fat substances from the waste water, and the particles of pollution of non-fat origin fall into the sediment. We consider this process in relation to the unit volume of wastewater $V$ (Fig. 1)

We assume that the pollution has the shape of a ball, and their number in the unit volume is $n_{1}$, with the diameter of these particles is $d_{1}$.

As a result of the impact of the phenomenon of acoustic cavitation on a particle with a diameter of $d_{1}$, the destruction of the shell from contamination of non-fat origin occurs. 


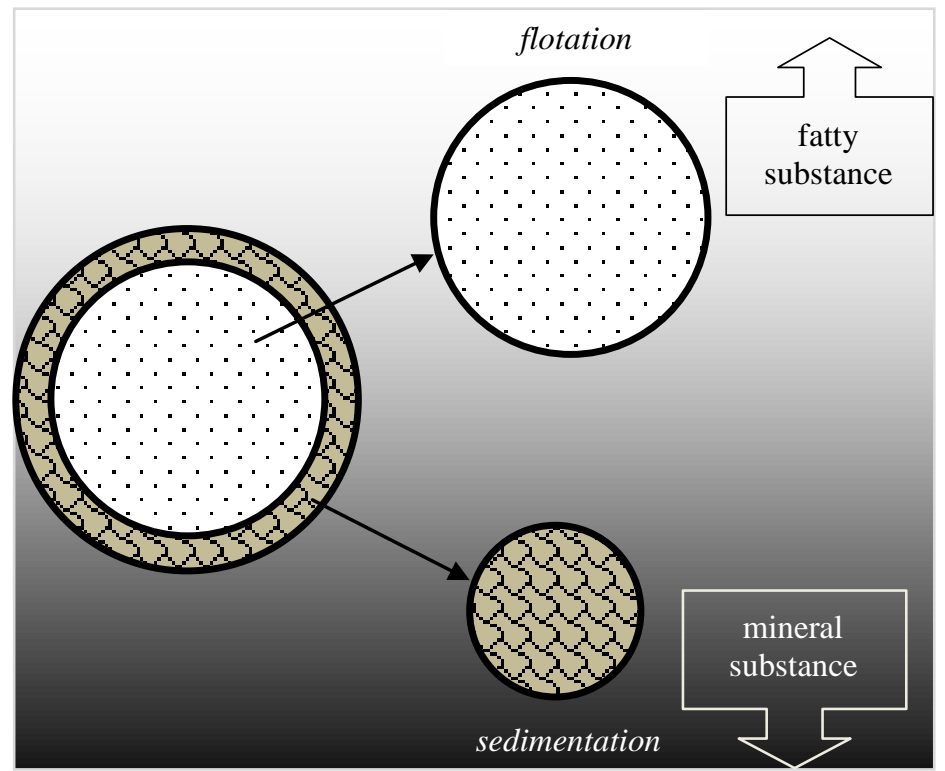

Fig. 1. Model of separation of fat and non-fat particles in a liquid medium under the action of ultrasonic field cavitation.

Fat particles of diameter $d_{2}$ float in water, and particles of pollution precipitate, and their diameter is $\left(d_{1}-d_{2}\right)$ [22].

If the number of particles of contamination per unit volume of waste water equal to $n_{1}$, then the amount of pollution associated with the fat is

$$
V_{1}=\frac{\pi \cdot d_{1}^{3}}{6} \cdot n_{1}
$$

where $V_{1}$ is the volume of all contaminants at the initial moment of separation.

We believe that under the influence of acoustic cavitation, all fat particles are released from impurities and their number is $n_{2}$, then the volume of fat particles separated from the impurities will be

$$
V_{2}=\frac{\pi \cdot d_{2}^{3}}{6} \cdot n_{2}
$$

where $V_{2}$ is the volume of fatty substances separated from contaminants.

The products of non-fat impurities precipitate, and the size of the precipitating particles will be equal to $\left(d_{1}-d_{2}\right)$ and they will occupy the volume of

$$
V_{3}=\frac{\pi \cdot\left(d_{1}-d_{2}\right)^{3}}{6} \cdot n_{1} .
$$

where $V_{3}$ is the volume of non-fat pollution.

Taking into account formulas $(11,12,13)$ volume of contaminants by types, you can write the balance equation of contamination of the waste water in perfect conditions, in compliance with all adopted assumptions.

Thus, 


$$
V_{1}=V_{2}+V_{3}
$$

in the left part of the equation (14) the volume of all pollution at the initial moment of separation, and in the right - the sum of the volumes obtained under the action of acoustic cavitation.

Substitute in this equation the expression $(11,12,13)$ and express the amount of pollution through the size of the corresponding particles

$$
\frac{\pi \cdot d_{1}^{3}}{6} \cdot n_{1}=\frac{\pi \cdot d_{2}^{3}}{6} \cdot n_{2}+\frac{\pi \cdot\left(d_{1}-d_{2}\right)^{3}}{6} \cdot n_{1},
$$

by converting and dividing all parts of the equation (15) into the first term we obtain

$$
1=\frac{n_{2}}{n_{1}} \cdot \frac{d_{2}^{3}}{d_{1}^{3}}+\frac{\left(d_{1}-d_{2}\right)^{3}}{d_{1}^{3}} .
$$

Denote by $N$ the concentration of fat substances in the volume unit, and $\Delta N-$ the concentration of fat substances released from the volume of wastewater. Make the proportion

$$
\frac{n_{2}}{n_{1}}=\frac{\Delta N}{N}=y
$$

where $y=f\left(n_{2} / n_{1}, \Delta N / N\right)$.

Substitute the expression (17) in equation (16) and write down

$$
y=\frac{1-\frac{\left(d_{1}-d_{2}\right)^{3}}{d_{1}^{3}}}{\frac{d_{2}^{3}}{d_{1}^{3}}},
$$

opening the brackets, we obtain (18)

$$
y=3 \frac{d_{1}}{d_{2}}-3\left(\frac{d_{1}}{d_{2}}\right)^{2}+1 .
$$

This equation-is the degree of change in the particle size when exposed to an ultrasonic field. This value depends on the destructive effect of acoustic cavitation. Therefore, we denote the action factor of the ultrasonic field on the system "liquid-solid" through $\mathrm{x}$, and substitute in the expression (19) in place $\frac{d_{1}}{d_{2}}$ we obtain an equation that characterizes the dependence of the release of fat substances from wastewater using an ultrasonic field.

The transformation of equation (19) yields the final result

$$
y=b \cdot x-a \cdot x^{2}+1,
$$

where $a, b$ are constants.

Analysis of equation (20) shows that $y=f(x)$ is a quadratic function. 


\section{Solutions}

1. Analysis of the effect of the ultrasonic field in the cavitation mode on the substance showed that the use of this method for the release of fatty substances from wastewater will intensify the purification process.

2. In theoretical calculations of the release of fatty substances from wastewater using an ultrasonic field made assumptions-pollution have the shape of a ball, which is dominated by fatty substances on the surface of the ball - mineral impurities.

3. The use of ultrasound in cavitation mode increases the concentration of fatty substances, their ascent due to the destruction of the outer shell of minerals.

\section{Conclusions}

1. The developed model and calculation method allow us to link the effect of cavitation on the purification effect by the function $y=f(x)$ according to the obtained equation $y=b \cdot x-$ $a \cdot x^{2}+1$.

2. The present equation of the model of the ultrasonic field effect in the cavitation mode on the substance allows to predict the effectiveness of this method of purification. The numerical value of the constants of the theoretical equation is obtained as a result of experimental studies.

\section{References}

1. I. P. Golyamina, Little Encyclopedia of Ultrasound (Soviet encyclopedia, Moscow, 1979)

2. Physics and technology of powerful ultrasound, Edited by L. D. Rosenberg, vol. 1 - 3 (Science, Moscow; 1967)

3. P. D. Vikulin, Physico-chemical manifestations of the acoustic field in water conditioning technologies (ASV, Moscow, 2004)

4. G. Flin, Physical acoustics: Translation from English (Mir, Moscow, 1967)

5. G. A. Kardashev, Physical methods of intensification of processes of chemical technology (Chemistry, Moscow, 1990)

6. R. Baldaev, Applications of ultrasound (Publishing House "Technosphere", Moscow, 2006)

7. M. G. Sirotyuk, Acoustic cavitation (Science, Moscow; 2008)

8. I. E. Elpiner, Biophysics of ultrasound (Nauka, Moscow, 1973)

9. M. A. Margulis, Successes of physical sciences 170 (3) 263-287 (2000).

10. V. M. Fridman, Ultrasonic chemical apparatus (Mashinostroenie, Moscow, 1967)

11. M. A. Mironov, Acoustic J. 23 (5) 825-830 (1977)

12. T. G. Leighton, The Acoustic Bubble (Academic Press, London, 1994)

13. V. N. Khmelev, A. V. Shalunov, A. V. Shalunov, Ultrasonic spraying of liquids (ASTU Publishing, Biysk, 2010)

14. S. P. Zubrilov, Physicochemical aspects of ultrasonic activation of astringent solutions. Doctoral dissertation abstract (Leningrad Institute of Water Transport, Leningrad, 1975).

15. V. A. Shutilov, Fundamentals of ultrasound physics (Leningrad University, Leningrad, 1980) 
16. S. P. Kundas and others, Ultrasonic processes in the production of electronic products. Vol.2 (BestPrint, Minsk, 2003)

17. Edited by B. A. Agranata, Ultrasonic technology (Metallurgy,Moscow, 1974).

18. V. N. Khmelev, Polzunovsky Herald 2/1 154-159 (2012)

19. I. M. Fedotkin, I. S. Guliy, Cavitation, cavitation technique and technology, their use in industry (theory, calculations and designs of cavitation devices). Part 1 (Poligrafkniga, Moscow, 1997)

20. N. V. Dezhkunov, T. G. Leighton, Engineering and Physics Journal 77 (1) $45-51$ (2004)

21. P. D. Vikulin, Development of the processes of separation of highly concentrated woolwater waste water by ultrasound. Dissertation, Candidate of Technical Sciences (MISI, Moscow, 1985)

22. E. V. Alekseev, Fundamentals of flotation wastewater treatment technology (ASV, Moscow, 2009) 